\title{
Org-Coursepack: A Modular and Reusable Teaching Materials Template in Org-mode
}

\author{
Joon $\mathrm{H} . \mathrm{Ro}^{1}$ and Jae-Eun Namkoong ${ }^{2}$
}

DOI: $10.21105 /$ jose. 00034

\section{Software}

- Review c⿻一𠃋

- Repository ca

- Archive cto

Submitted: 23 July 2018

Published: 15 January 2019

\section{License}

Authors of papers retain copyright and release the work under a Creative Commons Attribution $4.0 \mathrm{ln}$ ternational License (CC-BY).
1 Tulane University 2 University of Nevada, Reno

\section{Summary}

The Org-Coursepack provides a template for developing and managing teaching materials using Org mode, a major mode in GNU Emacs. The template is designed to be selfexplanatory since the documentation for it consists of a sample course developed with this template.

\section{Statement of Need}

Org-Coursepack started as an attempt to solve challenges authors experienced when creating and managing teaching materials. The key benefits of using the template as an instructor are summarized below.

First, sharing materials across courses becomes more effective and efficient with the template than with traditional means of creating lecture slides (e.g., PowerPoints or Beamer) because the template relies heavily on the file inclusion functionality of Org mode; thus, it is designed to be as modular as possible. As a result, a course topic can be used across multiple sections of the same course or across multiple courses, which means improvements made to one lecture file will automatically propagate across all sections and courses covering the topic, reducing redundancy and inconsistency. Thus, Org-Coursepack would be especially useful for an instructor with multiple courses that share common course materials.

Second, the flexible export system and output-specific export options in Org mode allow instructors to generate outputs in multiple formats. Any course-related material, such as syllabi, exam reviews, or lectures, can be easily exported in the form of slides (e.g., via reveal.js or Beamer backends) or handouts (e.g., via LaTeX or reStructuredText backends) while maintaining consistent content regardless of format. This allows instructors to create properly formatted, document-like handouts for their lectures, offering better presentation and legibility compared to scaled-down slides. In addition, instructors can choose to selectively present certain components (e.g., images, charts, or notes) depending on the output format. These features eliminate the need for instructors to maintain separate files for different output formats.

Third, the template contains utility functions written in Emacs Lisp, shortcuts to Org mode functions, and basic class structures, all of which contribute to automating a variety of instruction-related tasks. The features include, but are not limited to, automatic class numbering and automatic creation of key content, such as course schedules for syllabi, summaries of class materials, and exam keys.

In sum, the template can improve instructors' efficacy by minimizing redundancy and automating various tasks. This is important because repetitive, tedious, tangential tasks and 
the psychological distractions associated with them can pull instructors' attention away from core instructional responsibilities. Removing such elements makes course preparation more seamless, and redirecting the newly found time and mental resources to positive educational outlets can enhance instructors' intrinsic motivation and creativity (Csikszentmihalyi, 2014).

We believe students are the ultimate beneficiaries of this approach since their overall learning experience can be enhanced through consistent, properly formatted, strategically presented course materials that are more engaging and easier to comprehend. Such materials are also easier to digest and review outside the classroom.

Two business courses related to marketing analytics, which share about $60 \%$ of their course materials, were successfully created and managed using this template. We have received positive feedback from students about the benefits of having well-organized, high-quality teaching materials, as shown by the following comments from students' course evaluations:

The slides are extremely helpful and I appreciate that because textbooks are expensive and unnecessary when the lecture material is of this caliber.

The teaching materials could not be any better. It was very helpful to expect a handout at the beginning of each class, one that was formatted identically to the others and was very clear about communicating the content of the lecture.

The documentation provided in the Org-Coursepack is designed to be straightforward so instructors with basic knowledge about Org mode can customize it to meet their unique needs, whether they are creating new course content or converting existing courses. Outside a traditional classroom setting, the tool may be used for a wide range of instructional purposes, such as employee training sessions, workshops, and consumer education. Wellorganized, effectively presented course materials can enhance fluency in self-regulated learning; thus, the template may be especially beneficial for online courses in which independent work and learnercontent interaction are critical to student satisfaction and success (Kuo, Walker, Schroder, \& Belland, 2014).

More broadly, Org mode is useful for not only instructors but also practitioners across a variety of fields. For instance, businesses can use the approach when documenting multiple, often overlapping products and services either for internal recording purposes or for presentations to external clients. It is also useful to those managing a myriad of legal or other administrative documents that often refer to one another or contain common elements. We hope the Org-Coursepack, and Org mode in general, will contribute to the productivity of both those who use it and those who are served by it.

\section{References}

Csikszentmihalyi, M. (2014). Flow And The Foundations Of Positive Psychology. doi:10.1007/978-94-017-9088-8

Kuo, Y. C., Walker, A. E., Schroder, K. E., \& Belland, B. R. (2014). Interaction, Internet self-efficacy, and self-regulated learning as predictors of student satisfaction in online education courses. Internet and Higher Education, 20, 35-50. doi:10.1016/j.iheduc.2013.10.001 